\title{
An Electrospray Ionization Mass Spectrometric Study of the Subunit Structure of the Giant Hemoglobin from the Leech Nephelopsis oscura
}

\author{
Brian N. Green \\ Waters Corporation, MS Technologies Center, Micromass UK Ltd., Manchester, UK \\ Serge N. Vinogradov \\ Department of Biochemistry and Molecular Biology, Wayne University School of Medicine, Detroit, \\ Michigan, USA
}

\begin{abstract}
The subunit structure of the giant, extracellular hexagonal bilayer (HBL) hemoglobin $(\mathrm{Hb})$ from the leech Nephelopsis oscura was investigated by electrospray ionization mass spectrometry (ESI-MS) employing a maximum entropy deconvolution of its complex, multiply charged ESI spectra. The denatured unreduced $\mathrm{Hb}$ consisted of three monomer globin chains (M), a1 = 16,535 Da, a2 =17,171 Da and a3 = 17,315 Da, five nonglobin linker chains, L1 = $24,512 \mathrm{Da}, \mathrm{L} 2=24,586 \mathrm{Da}, \mathrm{L} 3=24,979 \mathrm{Da}, \mathrm{L} 4=25,006 \mathrm{Da}$, and L5 $=25,566 \mathrm{Da}$ and two subunits of 32,950 Da and 33,125 Da. ESI-MS of the denatured, reduced $\mathrm{Hb}$ showed that the latter were disulfide-bonded heterodimers (D) of globin chains b1 $=16,322 \mathrm{Da}$ and b2 $=16,499$ Da with chain $\mathrm{c}=16,632 \mathrm{Da}$. Time-of-flight ESI-MS of the $\mathrm{Hb}$ at $\mathrm{pH} \mathrm{3.8,} \mathrm{4.5,} \mathrm{5.0,} 5.8$ and 7.0 revealed a distribution of charge states from $32^{+}$to $37^{+}$with masses decreasing from 211 to $208.5 \mathrm{kDa}$ with increase in cone voltage from 60 to $160 \mathrm{~V}$, indicating the presence of a subassembly comprising 12 globin chains. The subunit composition $6 \mathrm{M}+3 \mathrm{D}+12 \mathrm{~h}$, where $\mathrm{M}$ $=16993 \mathrm{Da}$ and $\mathrm{D}=33004 \mathrm{Da}$ are the weighted masses and $\mathrm{h}=616.5 \mathrm{Da}$, provides a calculated mass, $208.37 \mathrm{kDa}$ that is closest to $208.5 \mathrm{kDa}$. Our experimental findings are consistent with the bracelet model of HBL Hbs, verified by the recent low-resolution crystal structure of Lumbricus $\mathrm{Hb}$, wherein an HBL arrangement of 12 globin dodecamer subassemblies is tethered to a central complex of 36 linker chains for a total mass of $208.37 \times 12+24.94 \times 36=3398$ kDa. (J Am Soc Mass Spectrom 2004, 15, 22-27) (c) 2004 American Society for Mass Spectrometry
\end{abstract}

$\mathrm{T}$ The giant, extracellular hemoglobins (Hbs) and chlorocruorins (Chls) (containing an altered heme with a formyl substituting for the 3-vinyl group) found in terrestrial, aquatic, and marine annelids and in deep sea vestimentiferans and annelids share the following properties: A characteristic hexagonal bilayer (HBL) appearance and size in electron micrographs, approximately $30 \mathrm{~nm}$ in diameter and 20 $\mathrm{nm}$ in height, a sedimentation coefficient of about $60 \mathrm{~S}$ and an abnormally low iron content of $0.23 \mathrm{wt} . \%$ [1-3]. These giant complexes were found to have masses of $\sim 3500 \mathrm{kDa}$ and to consist of two types of polypeptide chains: heme-containing 16-17 $\mathrm{kDa}$ globin chains and non-globin, linker chains of 24-32 kDa containing 9 to 12 cysteines [4]. The amino acid sequences of the globin chains are clearly related to vertebrate and invertebrate

Published online November 19, 2003

Address reprint requests to Dr. S. N. Vinogradov, Department of Biochemistry and Molecular Biology, Wayne State University School of Medicine, Detroit, MI 48201, USA. E-mail: svinogra@med.wayne.edu globins [5] and they form disulfide-bonded dimer, trimer and tetramer subunits as well as noncovalent subassemblies, ranging from dimer to dodecamer [4].

Over the last decade, ESI-MS has been shown to be the method of choice for the characterization of proteins $[6,7]$ by providing highly accurate masses. In particular, in the case of heteromultimeric protein complexes, as exemplified by the HBL Hbs, ESI-MS provides an exquisitely accurate enumeration of the constituent chains and covalently bonded subunits, as well as a quantitative determination of free and disulfidebonded Cys residues [8]. Furthermore, the combination of nanoflow electrospray and time-of-flight (TOF) mass analyzers has recently allowed the investigation of the quaternary structure of large noncovalent protein complexes [9].

We describe here the results of ESI-MS and ESITOF-MS studies of the polypeptide chain and subunit composition and noncovalent subassemblies of the HBL $\mathrm{Hb}$ from the leech Nephelopsis oscura. 


\section{Experimental}

\section{Materials}

Live Nephelopsis oscura were obtained from St. Croix Biological (Stillwater, $\mathrm{MN}$ ). The $\mathrm{Hb}$ was prepared as described earlier for the leech Macrobdella decora, by rapid homogenization in the cold room, in $0.1 \mathrm{M}$ Tris.Cl buffer, $1 \mathrm{mM}$ EDTA at neutral $\mathrm{pH}$ in the presence of a protease inhibitor cocktail (no. 1697-498, Biochemical Division, Boehringer Mannheim Corp., Indianapolis, IN) [10]. The homogenate was immediately centrifuged at $\sim 20,000 \times g$ for $20 \mathrm{~min}$ at $4{ }^{\circ} \mathrm{C}$ to remove cellular debris; this step was repeated as many times as necessary until a clear solution was obtained. The supernatant was then centrifuged at $\sim 150,000 \times g$ for $1 \mathrm{~h}$ to pellet the $\mathrm{Hb}$, the pellet dissolved and centrifuged again. The final pellet was dissolved and stored in the Tris buffer in liquid nitrogen.

\section{Preparation of Samples for ESI-MS}

Initially, a stock solution of the native $\mathrm{Hb}$ was made in water to give a concentration of $20 \mathrm{mg} / \mathrm{ml}$. Working solutions of the native $\mathrm{Hb}$, actually denatured native $\mathrm{Hb}$, were $0.2 \mathrm{mg} / \mathrm{ml}$ in $50 \%$ aqueous acetonitrile containing $0.2 \%$ formic acid. Reduction was effected by diluting $5 \mu \mathrm{l}$ of the stock solution in $80 \mu \mathrm{l}$ of $10 \mathrm{M}$ aqueous urea, to which $10 \mu \mathrm{l}$ of $1 \mathrm{M}$ ammonium bicarbonate and $10 \mu \mathrm{l}$ of $0.1 \mathrm{M}$ DTT solutions were added. After incubating the resulting solution for 10 min at $37{ }^{\circ} \mathrm{C}, 200 \mu \mathrm{l}$ of $1 \%$ formic acid was added and the solution centrifuged to dryness in a $10 \mathrm{kDa}$ cut-off centrifugal filter device (Microcon YM-10, Millipore Corp., Bedford, MA). The mixture of reduced chains retained on the filter was dissolved in $300 \mu \mathrm{l}$ of $1 \%$ formic and centrifuged again to remove the urea and reagents. Finally, the reduced chains were dissolved in $60 \mu \mathrm{l}$ of $1 \%$ formic acid, $90 \mu \mathrm{l}$ water and $150 \mu \mathrm{l}$ acetonitrile to make the working solution for introduction into the ESI source of the mass spectrometer. Reduced and carbamidomethylated $\mathrm{Hb}$ was produced by adding $5 \mu \mathrm{l}$ of $0.2 \mathrm{M}$ iodoacetamide to a fresh preparation of the solution used to effect reduction as described above. This solution was incubated for $15 \mathrm{~min}$ at $37{ }^{\circ} \mathrm{C}$, diluted with $1 \%$ formic acid and centrifuged twice as described above to remove the urea and reagents. Finally, the derivatized chains were dissolved as above to make the working solution for introduction into the mass spectrometer.

Samples for analysis under native conditions were initially desalted using $10 \mathrm{kDa}$ cut-off centrifugal filter devices (Millipore, type YM-10) and finally diluted to a concentration of $0.5 \mathrm{mg} / \mathrm{ml}$ in HPLC grade water. Solutions $(\sim 200 \mu \mathrm{l})$ were thoroughly desalted by shaking them for about 1 min with $\sim 5 \mathrm{mg}$ of previously washed $(2 \times$ with water) mixed-bed ion exchange resin beads (AG 501-X8; Bio-Rad, Hercules, CA). Ammonium acetate was added to $10 \mathrm{mM}$ concentration and the $\mathrm{pH}$ adjusted with dilute ammonia or formic acid solution to obtain the desired $\mathrm{pH}$ in the range 3.8 to 7.0. Given the very low buffering capacity of ammonium acetate in the $\mathrm{pH}$ range $6-7$, the experimental values in this range are approximate.

\section{Electrospray Ionization Mass Spectrometry}

ESI-MS data of denatured $\mathrm{Hb}$ were acquired on a Quattro Ultima electrospray mass spectrometer (Waters Corp., Micromass UK Ltd., Manchester, UK), using a sample flow rate into the electrospray source of 5 $\mu \mathrm{l} / \mathrm{min}$; data were typically acquired over 5-10 min. Processing used a maximum entropy (MaxEnt) based approach [11, 12] incorporated as part of the Micromass MassLynx software suite supplied with the spectrometer. Mass scale calibration employed the multiplycharged series from horse heart myoglobin (M-1882, Sigma Chemical Co., St. Louis, MO), using a calculated mass of $16951.5 \mathrm{Da}$ [13], based on the following atomic weights: $\mathrm{C}=12.011, \mathrm{H}=1.00794, \mathrm{~N}=14.00674, \mathrm{O}=$ 15.9994 , and $S=32.066$ [14].

ESI-MS data under native conditions were obtained by introducing the desalted sample solutions at $4 \mu \mathrm{l}$ / min into the standard source of a LCT, TOF instrument (Waters Corp., Micromass UK Ltd.). Typical parameters were: Capillary, $3 \mathrm{kV}$; cone, 60-160 V; source temperature, $110^{\circ} \mathrm{C}$; extraction cone, $3 \mathrm{~V}$; RF lens, $400 \mathrm{~V}$. Data were accumulated for 5-20 min. Mass scale calibration used the $\mathrm{Cs}_{(n+1)} \mathrm{I}_{n}{ }^{+}$ions from separate introductions of CsI (1 mg/ml) in 50\% water/2-propanol. Mass assignments were made from the smoothed data using $\mathrm{m} / \mathrm{z}$ values measured at the highest points of the multiply charged peaks and assuming multiple protonation. Hence, mass $=n(m / z-\mathrm{H})$, where $n$ is the number of charges and $\mathrm{H}$ is the proton mass. The mass of a subassembly was expressed as the mean of 3-5 peak top values and a standard deviation (SD). The MaxEnt software was used initially to find the approximate mass of each subassembly and hence the charge on each multiply charged species. It was not used to establish the accurate mass, because it fits symmetrical Gaussian peak shapes to the experimental data and the experimental peak profiles were asymmetrical due to adduct formation.

\section{Results and Discussion}

Figure 1 shows the MaxEnt derived spectra of the acid-denatured (Figure 1a), denatured and reduced (Figure 1b), and denatured, reduced and carbamidomethylated (Figure 1c) Nephelopsis $\mathrm{Hb}$. Three groups of peaks were observed in Figure 1a: Monomer (M) globin chains a1-a3 at $\sim 17 \mathrm{kDa}$, nonglobin linker chains L1-L5 at $24-26 \mathrm{kDa}$, and two subunits D1 and D2 at $\sim 33 \mathrm{kDa}$. Three monomer globin chains were observed, a1 = $16,535 \mathrm{Da}, \mathrm{a} 2=17,171 \mathrm{Da}$, and a3 = 17,315 Da. Four of the five linker chains occur unmodified in ESI-MS of the native $\mathrm{Hb}$ (Figure 1a): $\mathrm{L} 1=24,512 \mathrm{Da}, \mathrm{L} 2=24,586 \mathrm{Da}$, 

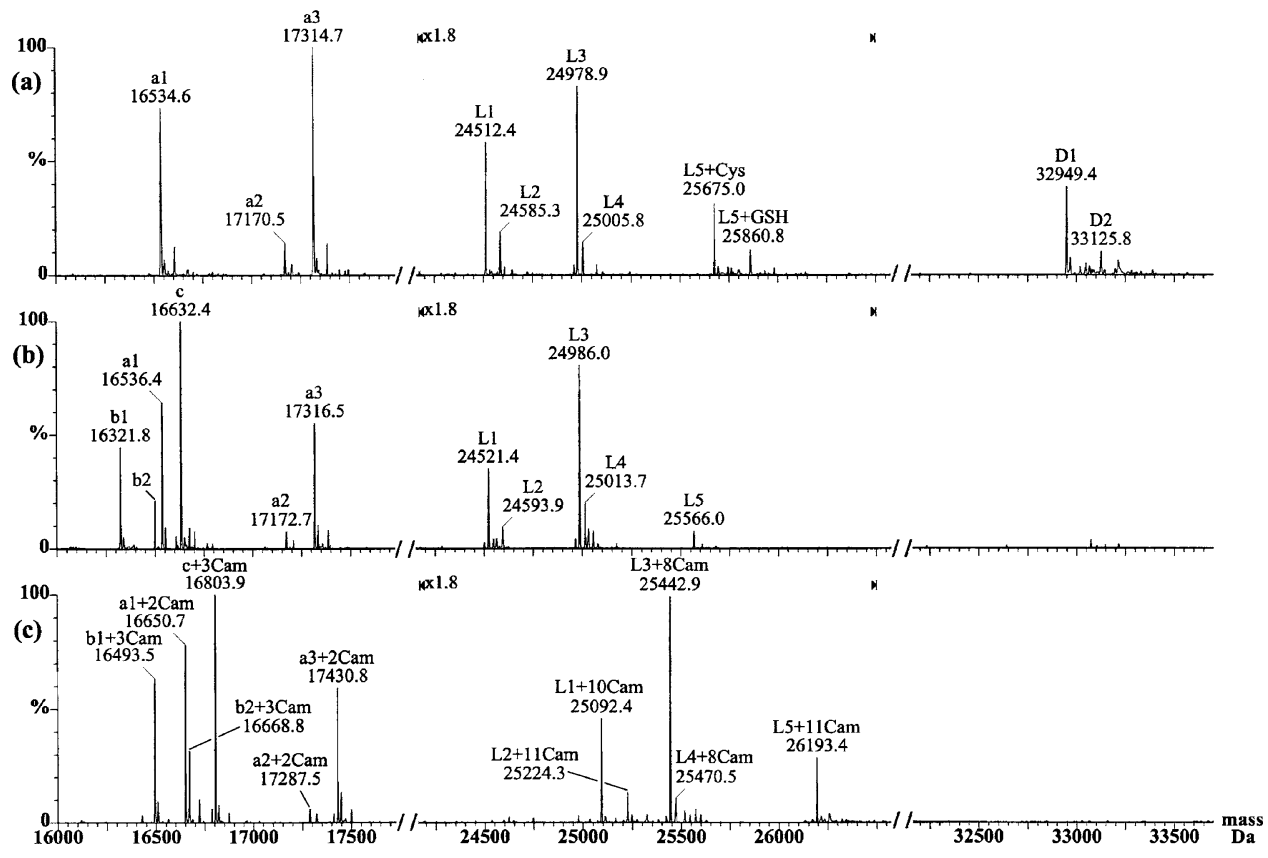

Figure 1. MaxEnt deconvoluted ESI-MS of Nephelopsis Hb: (a) Native acid-denatured; (b) reduced; (c) reduced and carbamidomethylated.

$\mathrm{L} 3=24,979 \mathrm{Da}$, and L4 = 25,006 Da. Linker L5 appears in monocysteinylated and monoglutathionylated forms. ESI-MS of reduced acid-denatured Nephelopsis $\mathrm{Hb}$ (Figure $1 \mathrm{~b}$ ) demonstrated that subunits D1 and D2 were disulfide-bonded heteromeric globin dimers, comprising chains $\mathrm{b} 1=16322 \mathrm{Da}$ and $\mathrm{b} 2=16499 \mathrm{Da}$ in combination with chain $\mathrm{c}=16632 \mathrm{Da}$, respectively, D1 $=\mathrm{b} 1+\mathrm{c}$ and $\mathrm{D} 2=\mathrm{b} 2+\mathrm{c}$. The spectrum of reduced and carbamidomethylated $\mathrm{Hb}$ shown in Figure 1c provides data on the presence and number of Cys residues in the polypeptide chains and subunits of the Hb. Table
1 lists the experimental masses of the globin chains and subunits of Nephelopsis $\mathrm{Hb}$, and the number of Cys residues present, derived by comparing the masses of the native, reduced and reduced and carbamidomethylated $\mathrm{Hb}$. Table 2 shows the self-consistency of the reported masses and Table 3 presents the available data on the relative intensities of the globin and linker subunits.

The ESI-MS of denatured Nephelopsis $\mathrm{Hb}$ illustrated in Figure 1 and the complete description of its constituent polypeptide chains and subunits summarized in

Table 1. The globin chains and subunits of Nephelopsis $\mathrm{Hb}^{\mathrm{a}}$

\begin{tabular}{|c|c|c|c|c|c|}
\hline Subunits & Native $^{\mathrm{b}}$ & Reduced $^{\mathrm{c}}$ & $\mathrm{Red} / \mathrm{cam}^{\mathrm{d}}$ & Corrected $^{\mathrm{e}}$ & Total Cys \\
\hline a1 & 16534.5 & 16536.4 & 16650.7 & 16536.6 & 2 \\
\hline a2 & 17170.5 & 16172.8 & 16286.8 & 16172.7 & 2 \\
\hline a3 & 17314.7 & 17316.5 & 17430.7 & 17316.6 & 2 \\
\hline L1 & 24512.3 & 24521.4 & 25092.5 & 24522.0 & 10 \\
\hline L2 & 24585.5 & 24594.1 & 25224.4 & 24596.8 & 11 \\
\hline L3 & 24978.9 & 25986.0 & 25443.0 & 24986.6 & 8 \\
\hline L4 & 25005.8 & 25013.5 & 25470.8 & 25014.4 & 8 \\
\hline L5 & ND & 25565.8 & 26193.7 & 25566.1 & 11 \\
\hline L5 + Cys & 25675.1 & ND & ND & ND & \\
\hline $\mathrm{L} 5+\mathrm{GSH}$ & 25860.4 & ND & ND & ND & \\
\hline D1 & 32949.5 & & & & 6 \\
\hline $\mathrm{D} 2$ & 33125.4 & & & & 6 \\
\hline b1 & & 16321.8 & 16493.6 & 16322.4 & 3 \\
\hline b2 & & 16498.6 & 16668.6 & 16497.6 & 3 \\
\hline c & & 16632.4 & 16803.81 & 16632.6 & 3 \\
\hline
\end{tabular}

aMasses in Da. Estimated errors: \pm 1 Da for a1-a3, b1, b2 and c; \pm 2 Da for L1-L5 and \pm 3 Da for D1 and D2. ND- not detected; GSH-glutathione. bMean of seven determinations.

${ }^{\mathrm{c}}$ Mean of two determinations of reduced $\mathrm{Hb}$.

dMean of four determinations for reduced and carbamidomethylated $\mathrm{Hb}$

' Corrected for carbamidomethylation (57.05 Da per Cys). Values are masses with Cys reduced.

fNumber of $\mathrm{Cys}=(\operatorname{Red} /$ Cam mass-Red mass $) / 57.05$ rounded to the nearest integer. 
Table 2. Self-consistency of mass assignments ${ }^{a}$

\begin{tabular}{lccr}
\hline Chain/subunit & $\begin{array}{c}\text { Number of } \\
\text { disulfide } \\
\text { bonds }\end{array}$ & $\begin{array}{c}\text { Calculated } \\
\text { mass }^{\text {b }}\end{array}$ & $\begin{array}{c}\text { Mass } \\
\text { difference }\end{array}$ \\
\hline \hline a1-2H & 1 & 16534.6 & 0.1 \\
a2-2H & 1 & 16170.7 & 0.2 \\
a3-2H & 1 & 17314.6 & -0.1 \\
L1-10H & 5 & 24511.9 & -0.4 \\
L2-11H & 5 & 24586.7 & 1.2 \\
L3-8H & 4 & 24978.5 & -0.4 \\
L4-8H & 4 & 25006.3 & 0.5 \\
L5-10H + Cys & $5^{\text {d }}$ & 25675.2 & 0.1 \\
L5-10H + GSH & $5^{\text {e }}$ & 25861.3 & 0.9 \\
D1 = b1 + c - 6H & 3 & 32949.0 & -0.5 \\
D2 $=$ b2 + c - 6H & 3 & 33124.2 & -1.2 \\
\hline
\end{tabular}

${ }^{a}$ Masses in Da.

${ }^{\mathrm{b}}$ Calculated from corrected mass in Table 1.

cCalculated mass-native mass from Table 1.

${ }^{\mathrm{d} C o m p r i s i n g}$ five intrachain disulfide bonds and cysteinylation (+119.1 Da) of the odd cysteine.

${ }^{e}$ Comprising five intrachain disulfide bonds and glutathionylation $(+305.3 \mathrm{Da})$ of the odd cysteine.

Table 1 , shows that it is composed of six globin chains with masses between 16,300 and $17,300 \mathrm{Da}$, all containing one intramolecular disulfide bond, three of which are monomeric and three of which have one additional Cys residue allowing them to form disulfide-bonded heterodimers. In addition, five nonglobin linker chains were observed with masses ranging from 24,500 to 25,600 Da. Linkers L1, L2, and L5 have five intramolecular disulfide bonds and L3 and L4 have four intramolecular disulfide bonds. Furthermore, linker L5 has an odd number of Cys residues (11), one of which appears to occur in cysteinylated and glutathionylated forms. The subunit composition of Nephelopsis $\mathrm{Hb}$ is in agreement with those of the leeches Macrobdella decora [15] and Haemopis sanguisuga [16] and is also found for the deep-sea hydrothermal vent polychaete, Riftia pachyptila [17].

These results illustrate the potency of the MaxEnt deconvolution software in disentangling the multiply charged data produced from complex protein mixtures by ESI-MS. Approximately twenty components, including adducts, were detected in each data set with intensities relative to the most intense component down to $\sim 4 \%$, corresponding to a useful dynamic range of $\sim 25: 1$. The precision of mass determination for the eleven subunits measured from the denatured native $\mathrm{Hb}$ (Table 1) when expressed in fractional mass terms was $< \pm 25 \mathrm{ppm}$ SD $(n=7)$. These data were acquired with independent calibration on three occasions over eight days. Table 2 illustrates the self-consistency of mass determination by comparing the mass of each subunit measured directly from the denatured $\mathrm{Hb}$ data with that calculated from the reduced mass derived from the reduced and carbamidomethylated data. The differences were all $<1.2 \mathrm{Da}(<50 \mathrm{ppm})$. The accuracy was not assessed independently by comparing experimental masses with sequence masses, because to our knowledge, none of the polypeptide chains in Nephelopsis have been sequenced. However, previous work with Glycera $\mathrm{Hbs}$ [18] indicates that the estimated errors for the globin chains (Table 1 footnote, $<60 \mathrm{ppm}$ ) were attained in this work.

Table 3 shows the relative intensities of the subunits obtained by ESI-MS from the denatured, reduced and reduced/carbamidomethylated $\mathrm{Hb}$. Clearly, in terms of relating abundance to intensity, these data are semiquantitative at best, since the ESI-MS sensitivities of different polypeptide chains are not necessarily the same. Comparing intensities should be restricted to similar chains, i.e., within the groups of globins or linkers or dimers. Bearing in mind these reservations, the relative proportions of chains $\mathrm{a} 1+\mathrm{a} 2+\mathrm{a} 3$ and chains $b 1+b 2+c$ correspond to the relative proportions of the monomer globins and the globins in the disulfide linked dimers, D1 and D2. An important conclusion that can be drawn from these data is that the

Table 3. Relative proportions of globin and linker subunits from the intensities of ESI-MS

\begin{tabular}{lccc}
\hline Chain & Denatured $^{\text {a }}$ & Reduced $^{\text {b }}$ & Reduced/cam $^{\text {b }}$ \\
\hline \hline a1 & 0.4 & $0.22(0.50)$ & $0.23(0.55)$ \\
a2 & 0.1 & $0.03(0.07)$ & $0.02(0.05)$ \\
a3 & 0.5 & $0.19(0.43)$ & $0.17(0.40)$ \\
D1 & 0.7 & - & - \\
D2 & 0.3 & - & - \\
b1 & - & 0.15 & 0.19 \\
b2 & - & 0.07 & 0.09 \\
C & - & 0.34 & 0.30 \\
L1 & 0.3 & 0.24 & 0.23 \\
L2 & 0.1 & 0.06 & 0.06 \\
L3 & 0.3 & 0.52 & 0.51 \\
L4 & 0.1 & 0.13 & 0.05 \\
L5 & 0.2 & 0.06 & 0.15 \\
(a1 + a2 + a3):(b1 + b2 + c) & - & $0.44: 0.56$ & $0.42: 0.58$ \\
\hline
\end{tabular}

${ }^{a}$ Only the relative intensities of chains a1-a3 are provided, since the volatilization of subunits D1 and D2 should be lower than that of the monomeric globins.

${ }^{\mathrm{b} N u m b e r s}$ in parentheses are the fractions $\mathrm{an} /(\mathrm{a} 1+\mathrm{a} 2+\mathrm{a} 3), \mathrm{n}=1,2,3$. 


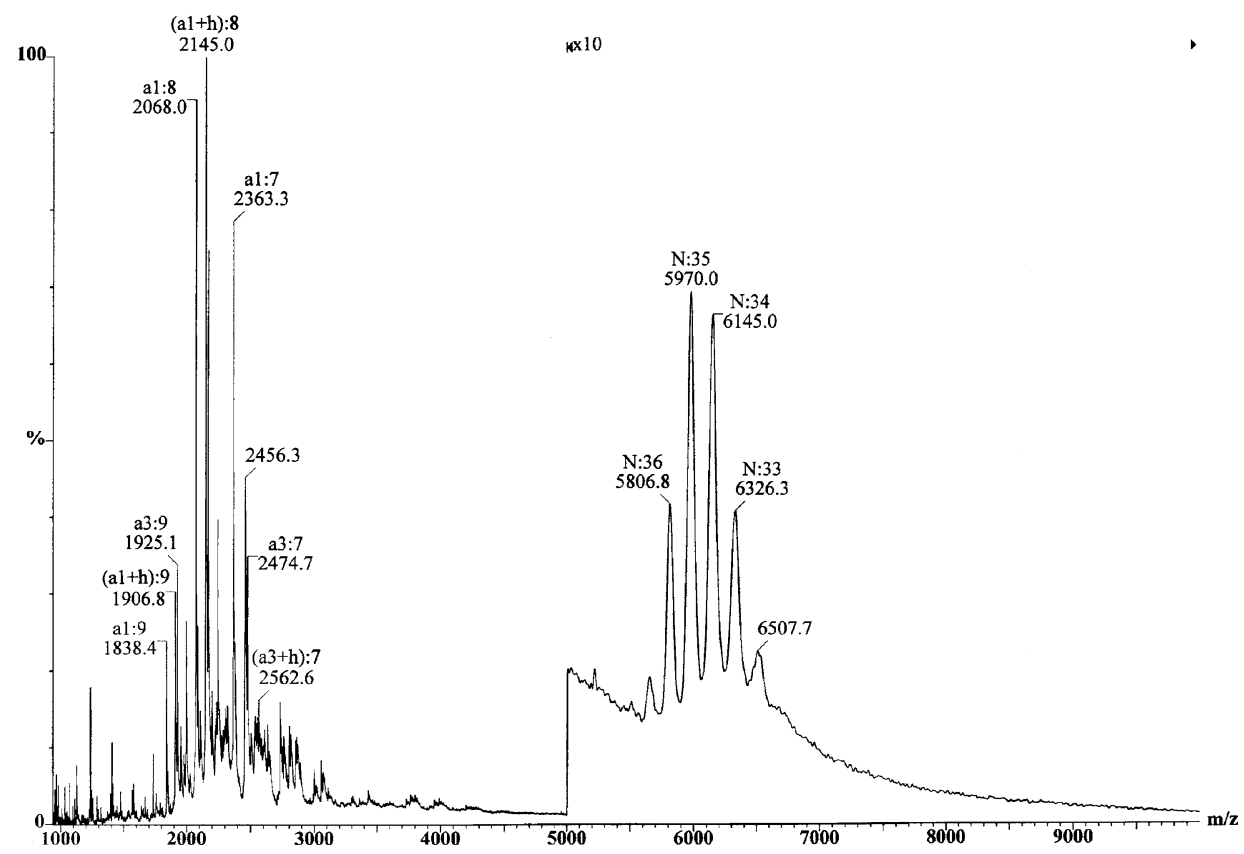

Figure 2. The ESI-TOF spectrum of an aqueous solution of Nephelopsis $\mathrm{Hb}$ at $\mathrm{pH} 7.0$ acquired with a $120 \mathrm{~V}$ declustering potential. Ions $(\mathrm{N})$ with 33-36 charges imply a mass of $208.9 \mathrm{kDa}$ assuming protonation.

proportions are approximately equal, as suggested by the bottom row in Table 3.

Figure 2 shows an ESI-TOF spectrum of Nephelopsis $\mathrm{Hb}$ obtained under native conditions from a solution at pH 7.0 with $120 \mathrm{~V}$ declustering potential (cone voltage). Present are species (N) that carry from 32 to 37 positive charges corresponding in this case to a sub-assembly mass of $208.9 \mathrm{kDa}$. Also present are species corresponding mainly to monomers with and without heme carrying 7-9 charges. All the results obtained from solutions at several values of $\mathrm{pH}$ ranging from 3.8 to7.0 and over the cone voltage range 60-160 showed similar spectra of the subassembly with masses between 208.4 to 211.0 $\mathrm{kDa}$. The observed mass varied inversely with declustering potential. In general, the variation was small, the maximum decrease in mass being $\sim 1.3 \%$ over the range $60-160 \mathrm{~V}$. At a given cone voltage, the observed masses of the subassembly were within experimental error of one another irrespective of the $\mathrm{pH}$. Table 4 shows how the mean mass of the subassembly $(n=3-5)$ varied with the declustering potential over the range $60-160 \mathrm{~V}$. The overall mass of the subassembly is dependent on the declustering potential, comparable to the effect observed earlier for similar subassemblies of Lumbricus and Arenicola HBL Hbs [19]. Declustering occurs when the positively charged protein ions are accelerated through a region that is intermediate in pressure between their generation at atmospheric pressure and analysis under high vacuum. This acceleration has the effect of imparting internal energy to the ions by collisions with the nitrogen gas in this region, causing them to lose weakly bound ligands at low potentials, followed by more strongly bound ligands as the potential is increased. Thus, at low declustering potentials, the mass tends to be higher than at high potentials.

The results in Table 4 show that the observed mass of the subassembly in Nephelopsis $\mathrm{Hb}$ tends towards a value slightly below $208.5 \mathrm{kDa}$ with increasing cone voltage. Also shown in Table 4 are the masses of globin dodecamer subassemblies calculated for different com-

Table 4. The observed masses of the globin subassemblies of Nephelopsis $\mathrm{Hb}$ at different declustering potentials and the calculated masses for possible dodecamer combinations

\begin{tabular}{|c|c|c|c|}
\hline Cone voltage, $\mathrm{V}$ & Observed masses, kDa & Calculated mass, $\mathrm{kDa}^{\mathrm{a}}$ & Subunit combinations \\
\hline 60 & $210.72 \pm 0.27$ & 210.28 & $10 M+D+12 h$ \\
\hline 80 & $210.07 \pm 0.28$ & 209.31 & $8 M+2 D+12 h$ \\
\hline 100 & $209.24 \pm 0.09$ & 208.33 & $6 M+3 D+12 h$ \\
\hline 120 & $208.87 \pm 0.07$ & 207.36 & $4 M+4 D+12 h$ \\
\hline 140 & $208.67 \pm 0.04$ & 206.38 & $2 M+5 D+12 h$ \\
\hline 160 & $208.6,208.5,208.4$ & & \\
\hline
\end{tabular}

${ }^{\mathrm{a}}$ Calculated using the weighted masses $\mathrm{M}=16,988 \mathrm{Da}$ and $\mathrm{D}=33,002 \mathrm{Da}$ and $\mathrm{h}=616.5 \mathrm{Da}$. 
binations of the intensity weighted mean masses of the monomers (M) and dimers (D), assuming one heme group $(\mathrm{h})$ is associated with each globin chain. The masses of M (16,988 Da), L (24,953 Da), and D (33,002 $\mathrm{Da})$ were calculated from the masses and relative intensities of the denatured native chains and subunits given in Table 1. It is obvious that the observed mass of the subassembly at the highest cone voltage corresponds best with the mass of the $6 \mathrm{M}+3 \mathrm{D}+12 \mathrm{~h}$ combination of globin subunits (208.33 kDa). This conclusion is supported by the approximately equal proportions of chains $\mathrm{a} 1+\mathrm{a} 2+\mathrm{a} 3$ relative to chains $\mathrm{b} 1+\mathrm{b} 2+\mathrm{c}$ indicated by the relative ESI-MS intensities in Table 3.

The direct observation of dodecamer globin subassemblies by ESI-TOF-MS in Nephelopsis $\mathrm{Hb}$ provides concrete evidence for their existence within these giant heteromultimeric complexes in solution. This result is in full accord with the "bracelet" model of the quaternary structure of HBL Hbs proposed earlier [20] and strongly supported by the recent low-resolution crystal structure of Lumbricus Hb [21]: An HBL complex of 12 dodecamer subassemblies tethered to 36 linker chains. Based on this model the expected mass of Nephelopsis $\mathrm{Hb}$ would be $208.33 \times 12+24.95 \times 36=$ $3398 \mathrm{kDa}$, well within the observed range of masses of HBL Hbs [4].

\section{References}

1. Vinogradov, S. N.; Shlom, J. M.; Kapp, O. H.; Frossard, P. Comp. Biochem. Physiol. B 1980, 67, 1-15.

2. Vinogradov, S. N.; Kapp, O. H.; Ohtsuki, In Electron Microscopy of Proteins, Vol III; M. Harris, J., Ed.; Academic Press: New York, 1982; pp 135-163.

3. Vinogradov, S. N. In Respiratory Pigments in Animals; Lamy, J.; Truchot, J-P.; Gilles, R., Eds.; Springer Verlag: Berlin, 1986; pp 9-20.
4. Lamy, J. N.; Green, B. N.; Toulmond, A.; Wall, J. S.; Weber, R. E.; Vinogradov, S. N. Chem. Rev. 1996, 96, 3113-3124.

5. Kapp, O. H.; Moens, L.; Vanfleteren, J.; Trotman, C. N. A.; Suzuki, S.; Vinogradov, S. N. Protein Sci. 1995, 4, 2179-2190.

6. Fenn, J. B.; Mann, M.; Meng, C. K.; Wong, S. F.; Whitehouse, C. M. Science 1989, 246, 64-68.

7. Mann, M.; Hendrickson, R. C.; Pandey, A. Annu. Rev. Biochem. 2001, 70, 437-473.

8. Green, B. N.; Hutton, T.; Vinogradov, S. N. In Methods of Molecular Biology, Vol. LXI; Chapman, J. R., Ed.; Humana Press: London, 1996; pp 279-294.

9. Rostom, A. A.; Robinson, C. V. Curr. Opin. Struct. Biol. 1999, 9 , 135-141.

10. Kapp, O. H.; Qabar, A. N.; Bonner, M. C.; Stern, M. S.; Walz, D. A.; Schmuck, M.; Pilz, I.; Wall, J. S.; Vinogradov, S. N. J. Mol. Biol. 1990, 213, 141-158.

11. Ferrige, A. G.; Seddon, M. J.; Jarvis, S. A. Rapid Commun. Mass Spectrom. 1991, 5, 374-379.

12. Ferrige, A. G.; Seddon, M. J.; Green, B. N.; Jarvis, S. A.; Skilling, J. Rapid Commun. Mass Spectrom. 1992, 6, 707-711.

13. Zaia, J.; Annan, R. S.; Biemann, K. Rapid Commun. Mass Spectrom. 1992, 6, 32-36.

14. IUPAC Commission on Atomic Weights and Isotopic Abundances. J. Phys. Chem. Ref. Data 1993, 22, 1571-1584.

15. Weber, R. E.; Malte, H.; Sharma, P. K.; Green, B. N.; Oliver, R. W. A.; Braswell, E. H.; Kuchumov, A. R.; Vinogradov, S. N. J. Mol. Biol. 1995, 251, 703-720.

16. Green, B. N.; Kuchumov, A. R.; Klemm, D. J.; Vinogradov, S. N. Int. J. Mass Spectrom. Ion Processes 1999, 188, 105-112.

17. Zal, F.; Lallier, F. H.; Green, B. N.; Vinogradov, S. N.; Toulmond, A. J. Biol. Chem. 1996, 271, 8875-8881.

18. Green, B. N.; Loo, J. A.; Walz, D. A.; Vinogradov, S. N. J. Protein Chem. 1999, 17, 85-97.

19. Green, B. N.; Bordoli, R. S.; Hanin, L. G.; Lallier F. H.; Toulmond, A.; Vinogradov, S. N. J. Biol. Chem. 1999, 274, 28206-2813.

20. Vinogradov, S. N.; Lugo, S.; Mainwaring, M. G.; Kapp, O. H.; Crewe, A. V. Proc. Natl. Acad. Sci. U.S.A. 1986, 83, 8034-8038.

21. Royer, W. E.; Strand, K.; van Heel, M.; Hendrickson, W. A. Proc. Natl. Acad. Sci. U.S.A. 2000, 97, 7107-7111. 